DE DE GRUYTER

OPEN

G

BULGARIAN ACADEMY OF SCIENCES

CYBERNETICS AND INFORMATION TECHNOLOGIES • Volume 14, Special Issue

Sofia • 2014

Print ISSN: 1311-9702; Online ISSN: 1314-4081

DOI: $10.2478 /$ cait-2014-0040

\title{
Safety Risk Management in Large Scale Engineering Based on Model Predictive Control
}

\section{Qiao Jianfeng, Wang Changfeng}

Economy and Management School of Beijing University of Posts and Telecommunications, Beijing China 100876

Email: Jianfeng_qiao@126.com

\begin{abstract}
Safety risk during the construction of a Large Scale Engineering (LSE) project can be avoided through safety education, following a correct procedure and using an engineering method. In order to ensure that LSE works in a safe state, some parameters need to be restricted to a certain range. Besides, in order to apply the former control for the predictive values, a Model Predictive Control (MPC) theory is suggested to be used for safety risk controlling. We share a MPC work flow and detail calculation steps of the rolling optimization, feedback correlation and constraint control, and a mean time gradient decent method is used during the optimization calculation iteration steps. At the end, regarding the resource allocation issue in the ship-lift engineering construction area of "Three Gorges" we verify how MPC works; and the numeric results show MPC's efficiency. We can see that MPC might have important future in safety risk management of LSE, because as long as the model can be predictive, regardless of an accurate mathematical model of the system, MPC can control a dynamic and uncertain system very well, which is also a characteristic of the safety risk of LSE.
\end{abstract}

Keywords: Large Scale Engineering; safety risk; resource allocation; model predictive control. 


\section{Introduction}

Large Scale Engineering (LSE) [1] plays a very important role in human society. It is usually required to satisfy numerous competing functionality requirements, such as quality, performance, safety, economic concern, environmental impact, and so on. Due to the multi-objective of LSE, modem optimal control theory is applied into it, for example:

In 2006, in USA, Jeff Thielman pointed out that LSE needed to solve multiple, competing functionality requirements and provided a promising approach to efficiently evaluate and optimize a large-scale engineering system against multiple design objectives [2]. A systematic methodology is presented for applying Suh's axioms to evaluate and optimize large scale engineering systems.

In 2013, in Canada, Menesi Wail pointed out that LSE is challenged by many constraints, and Constraint Programming (CP) is used as an advanced mathematical technique that suits the schedule optimization problems [3]. Finally, a practical CP optimization model has been developed to resolve both the deadline and resource constraints simultaneously in LSE.

\subsection{LSE safety issue}

Obviously, the large scale engineering project is a complex giant integrated system, and the construction process is very uncertain, therefore, it often leads to safety risks of major disaster accidents, resulting in huge economic losses to the state property, and a large number of personnel casualties. The safety risk management process must first ensure that an adequate number of personnel is legally appointed to manage the safety and that employees are trained and understand the importance of job site safety.

There are several causes of safety incidents which are already identified by researchers, such as "lack of knowledge and training", "lack of quality, environmental, health and safety budget", "Overemphasis on speed of construction rather than quality, environmental, health and safety conditions" and so on [4]. Generally three kinds of methods are usually adopted in the solution of the safety risk, including safety education, following a correct procedure and an engineering method. Until now the researchers mainly focus on hazard identification and risk assessment [5, 6]. But risk control is also a very important process, and more quantitative, systematic, optimal methods must be introduced into safety risk management to give detailed scientific analysis of each safety risk factor. An optimal control algorithm begins to be applied in safety risk management [7], but it is offline optimization based on overall system performance. LSE is a dynamic system and it needs online optimization and must be more flexible to system changes, so that Model Predictive Control (MPC) must be considered, which is already used in the industrial processes in chemical plants and oil refineries since the 1980-ies.

\subsection{MPC in safety}

In order to ensure LSE work in a safe mode, some parameters need to be restricted to a certain range, such as: 
- maximum operating temperature,

- maximum loading capacity,

- maximum bearing pressure.

This means that the safety risk issue is a constraint control issue and due to the uncertainty of the model and the dynamic character of the system, MPC begins to be applied in some areas:

In 2011, in UK, Mietek A. Brdys has considered the design of Robustly Feasible Model Predictive Controller which achieves recursive robust feasibility of the generated control actions when applied to nonlinear network systems with a state constraint (safety zone) under bounded disturbances (see [8]). Its performance is illustrated by application to the optimizing control of hydraulics in Drinking Water Distribution System.

In 2013, in Norway, Torstein I. B. has introduced safety constraints based on fault scenarios and an economic model predictive control was used to control a plant into a fault-tolerant state to make sure that it controls the states to a state set, where the plant is recoverable if faults occur (see [9]). The control systems consider safe and energy efficient vessels with hybrid power plants.

In 2013, in USA, John M. Carson has built a reactive safety mode into a robust model predictive control algorithm for uncertain nonlinear systems (see [10]). The reactive safety mode, if initiated, contains closed-loop states within an invariant set about a desired safety reference for the whole time. The research is motivated by a vehicle control-algorithm design.

Based on the current research, MPC starts to resolve the safety issue in recent years and it is a new research area. In this paper we take a discrete system as an example and show how the safety issue can be solved by MPC with the help of detail steps. Generally, MPC algorithm is protected by a patent or commercial business [11], but we share a detailed MPC working flow including rolling optimization, feedback correlation and constraint control. Taking into account the resource allocation in ship-lift engineering construction area in "Three Gorges”, we share the calculation results and finally they show the efficiency of MPC.

\section{Safety issue of a discrete system}

There are lots of discrete systems in a large scale engineering project and generally they can be represented as given in Fig. 1.

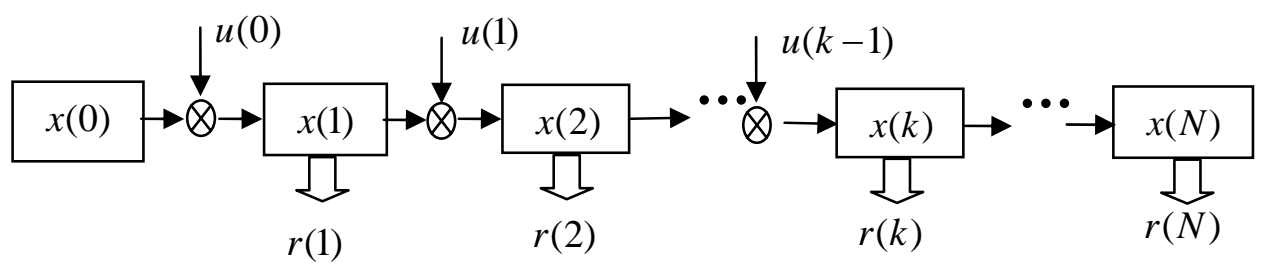

Fig. 1. A discrete system 
In the diagram $x(k)$ represents the current state of the system at $k$ stage, then there is a state vector sequence

$$
x(1), x(2), \ldots, x(k), \ldots, x(N) ; k=1,2, \ldots, N .
$$

$x(0)$ represents the initial state of the system; $x(N)$ represents the end state of the system.

Let $u(k)$ represents the control variant with respect to $k+1$ stage. This value represents the changes between $x(k)$ stage and $x(k+1)$ stage. Then there is a control vector sequence

$$
u(0), u(1), \ldots, u(k), \ldots, u(N-1) ; \quad k=0,1,2, \ldots, N-1 .
$$

Let $r(k)$ represents the project's demand for $x(k)$ at $k$ stage. The state sequence is as known:

$$
r(1), r(2), \ldots, r(k), \ldots, r(N) ; \quad k=1,2, \ldots, N .
$$

In general, we can get the requirement of $r(k)$ by the project plan. Based on the above assumptions, the system state at each stage is subject to the difference equations as follows:

$$
x(k+1)=x(k)+u(k) ; k=0,1,2, \ldots, N-1 .
$$

The equations show that at the given state $x(0)$ we can get a state sequence $x(k)$ by adjusting to the control sequence $u(k)$.

To meet the safety requirements, the dynamic system must generally satisfy the constraints:

$$
\left\{\begin{array}{l}
u_{\min } \leq u(k) \leq u_{\max }, \\
x_{\min } \leq x(k+1) \leq x_{\max },
\end{array} \quad k=0,1,2, \ldots, N-1 .\right.
$$

Take one safety factor "Overemphasis on speed of construction rather than quality, environmental, health and safety conditions" as an example: the project request $x(k)$ - the more the better, so that the project can speed up the schedule with more resources, while the safety condition requires some constraints like (2). This kind of a safety issue has the characteristics given below:

- The safety requirement not only requests the current state to meet the constraint, but also requests the further state to meet the constraint.

- It is an optimization issue that means the performance equation not only considers the project speed, but also needs to consider safety conditions.

- It is a dynamic system and there is unexpected disturbance.

MPC is an advanced method of process control which is generally intended to represent the behavior of complex dynamical systems. And the above safety issue can be resolved by MPC. 


\section{MPC model}

Consider the following discrete-time state-space system:

$$
\left\{\begin{array}{l}
x(k+1)=A x(k)+B u(k), \\
y(k+1)=C x(k+1),
\end{array} \quad k=0,1,2, \ldots, N-1,\right.
$$

subject to the input and output constraints

$$
\left\{\begin{array}{l}
u_{\min } \leq u(k) \leq u_{\max }, \\
y_{\min } \leq y(k+1) \leq y_{\max }
\end{array}, \quad k=0,1,2, \ldots, N-1\right.
$$

At time step $k$, the model is used to predict a series of $P$ future outputs of the system up to time $k+P$, that is

$$
\hat{y}_{P M}(k)=\hat{y}(k+p \mid k) ; \quad p=1,2, \ldots, P .
$$

These predictions are used to calculate $M$ optimal future input moves, $u(k+m \mid k)$ for $m=0,1, \ldots, M-1$. The parameters $P$ and $M$ are referred to as the prediction horizon and the control horizon respectively. The process target is to follow a desired reference $r_{P}(k)$ as closely as possible, shown in Fig. 2.

The minimization criterion for the computation of the optimal future moves is usually a quadratic cost function of the difference $\left(\varepsilon_{1}, \varepsilon_{2}, \ldots, \varepsilon_{P}\right)$ between the predicted output signal and the desired reference. This criterion can also include the control moves $u(k+m \mid k)$ in order to minimize the control effort.

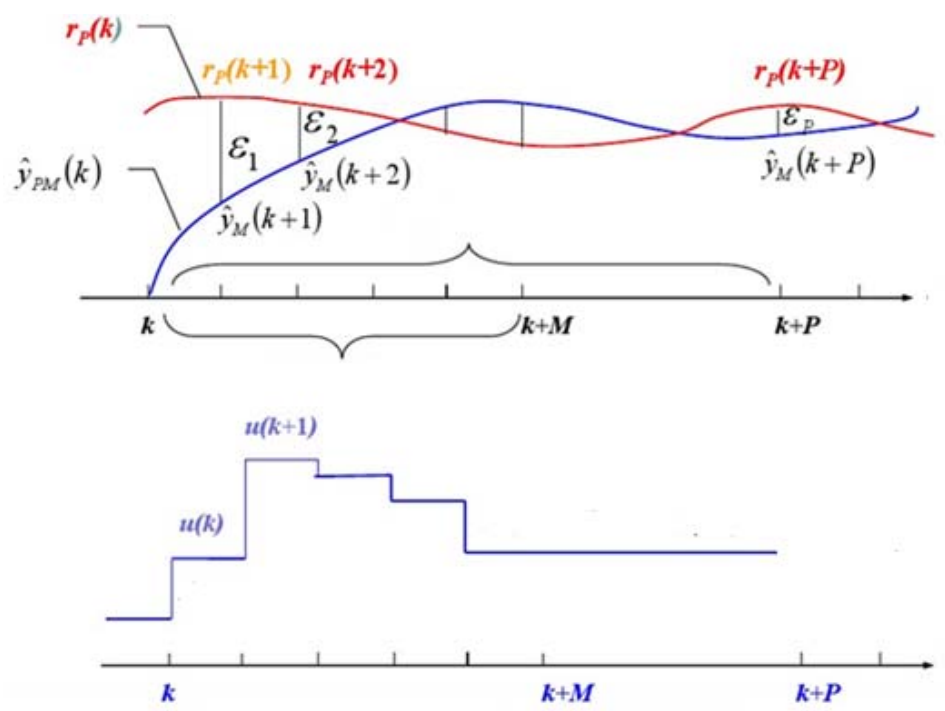

Fig. 2. MPC scheme

A typical objective function has the form

(5) $J_{P}(k)=\sum_{p=1}^{P} w_{p}^{y}\left[\hat{y}_{P M}(k+p) \mid k-r_{P}(k+p)\right]^{2}+\sum_{m=0}^{M-1} w_{m}^{u}[u(k+m)]^{2}$, 
subject to

$$
\left\{\begin{array}{l}
u_{\min } \leq u(k+m) \leq u_{\max } ; \quad m=0,1, \ldots, M-1 \\
y_{\min } \leq \hat{y}_{P M}(k+p) \leq y_{\max } ; \quad p=1,2, \ldots, P
\end{array}\right.
$$

where $w_{p}^{y}$ are the weight coefficients reflecting the relative importance of $\hat{y}_{P M}(k+p) \mid k ; w_{m}^{u}$ are the weighting coefficients penalizing relative big changes in $u(k+m)$.

\section{MPC theory}

There are three core parts in MPC that are rolling optimization, feedback correlation and constraint control, shown in Fig. 3.

- For rolling optimization, only $u(k)$ is the input to the system at the $k$ stage. Others, $u(k+1), \ldots, u(k+M-1)$ work with $u(k)$ to optimize the objective function and constrained by a safety condition. At $k+1$, re-calculate again.

- For feedback correlation, $y(k+1)$ is used, not only for a prediction model to update the prediction model if there is an error compared to $\hat{y}_{P M}(k+1) \mid k$, but also takes as a real input MPC for the next stage optimization.

- For a constraint, which is not only valid for current calculation of $u(k)$ and $y(k+1)$, but also valid for $u(k+1), \ldots, u(k+M-1)$ and $y(k+2), \ldots, y(k+P)$. This means that MPC safety control considers also the future control which is very important, because earlier warning and earlier control is considered.

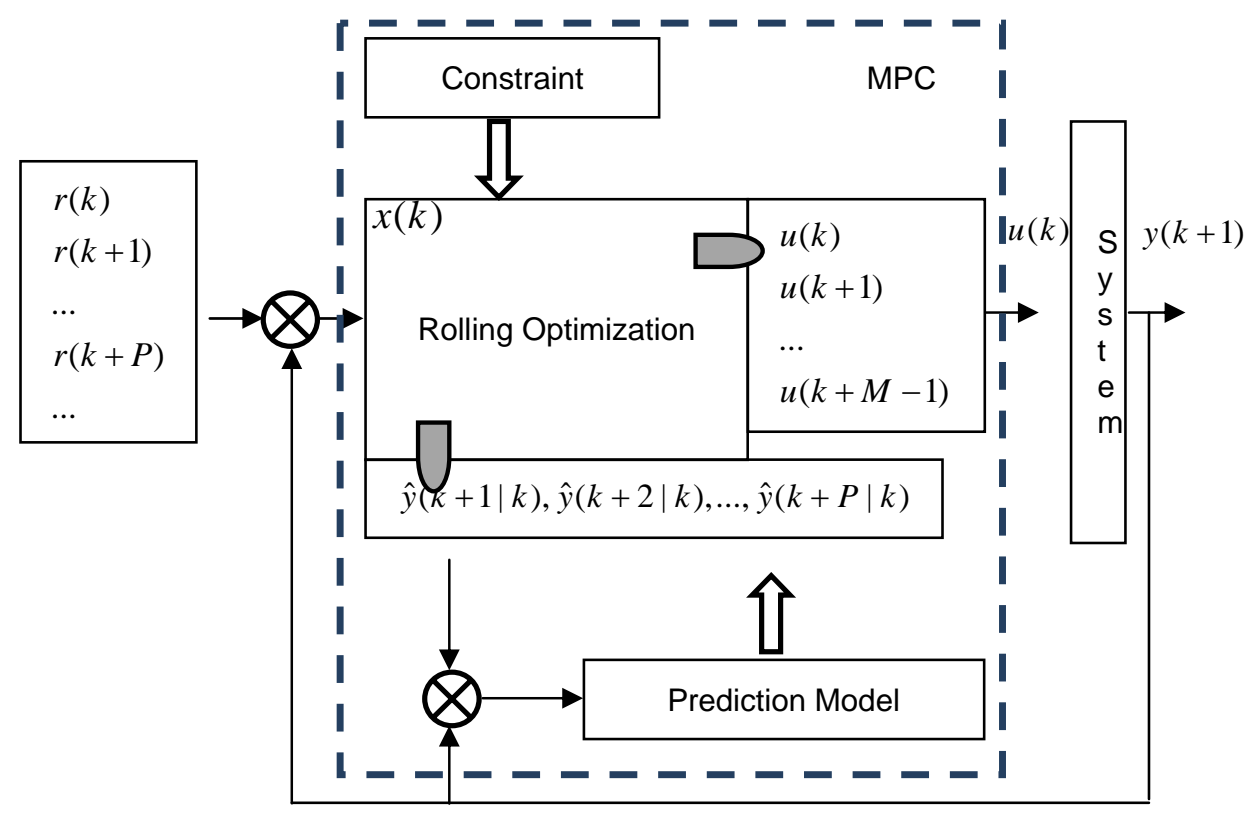

Fig. 3. MPC work flow 


\subsection{Optimization control}

MPC optimization control contains three main parts: the predictive model, the optimization calculation method and the rolling optimization.

\section{(1) Predictive model}

Use predictive modeling techniques to predict future outcomes. Considering (1), if $y(k+1)=x(k+1)$, (3) can be expressed as

$$
x(k+1)=x(k)+u(k) .
$$

Generally, the control predictive series is

$$
u(k+p)=u(k+M-1) ; p=M, M+1, \ldots, P-1 .
$$

However, based on Equation (1), this kind of prediction is meaningless, so we assume $P=M$ for a discrete system discussed in this paper. This means that at stage $k$, the control horizon series is

$$
u(k+0), u(k+1), \ldots, u(k+P-1) .
$$

The prediction horizon series is

$$
\hat{x}(k+1), \hat{x}(k+2), \ldots, \hat{x}(k+P) \text {. }
$$

\section{(2) Optimization calculation}

Simply assume

$$
w_{p}^{y}=A ; p=1,2, \ldots, P ; \quad w_{m}^{u}=B ; \quad m=1,2, \ldots, M-1 .
$$

The objective function can be updated into

$$
J_{P}(k)=\sum_{p=0}^{P-1} A\left[x(k+p)+u(k+p)+\sum_{p=0}^{P-1} B[u(k+p)]^{2} .\right.
$$

The above optimization issue can be resolved by a gradient descent method [7].

\section{(3) Rolling optimization}

At $k+1$ stage, after getting new measurements, repeat the optimization. It is also called "Rolling horizon".

\subsection{Feedback control}

In real applications, the feedback correction is very important. MPC must use a real feedback to give optimization control, not a predictive one.

If $\hat{x}(k+1) \neq x(k+1)$, the latter means a real feedback. Then at $k+1$ stage optimization control, MPC must use $x(k+1)$, not $\hat{x}(k+1)$. For a non-linear system, it uses the error to adjust the predictive model.

\subsection{Constraint control}

In (5) the constraint has not been considered. There are two kinds of constraints in this case. One is the output, that is

$$
x_{\min } \leq \hat{x}(k+p) \leq x_{\max } ; p=1,2, \ldots, P .
$$

The other one is the control variant 


$$
u_{\min } \leq u(k+p) \leq u_{\max } ; p=0,1, \ldots, P-1 .
$$

This also means

$$
u_{\min } \leq U^{M+1} \leq u_{\max }
$$

where $U^{M+1}=U^{M}-\alpha G^{M}$ and

$$
G^{M}=\partial J_{P}(k) / \partial u(k)
$$

is a descent gradient vector.

\section{Experimental study}

“Three Gorges” Project on Yangtze River is an important large scale engineering project in China. The safety risk is the most important concern for the government. Lots of researchers point out the safety risk during the construction and share the migration control strategy [12].

Take the ship-lift engineering construction area as an example. The project manager requires the workers numbers to be weekly the following, shown in Table 1.

Table 1. Reference value

\begin{tabular}{|c|c|c|c|c|c|c|c|c|c|c|c|c|}
\hline Ref. & W1 & W2 & W3 & W4 & W5 & w6 & W7 & W8 & W9 & W10 & W11 & W12 \\
\hline Reference & 11 & 24 & 33 & 7 & 26 & 19 & 18 & 21 & 37 & 16 & 31 & 25 \\
\hline
\end{tabular}

However, due to the narrow complex construction environment and the complexity of technical communication, we take a safety constraint:

- If $\hat{x}(k+p) \leq 30$ the maximum workers number must not exceed 30 due to the narrow environment and the requirement to keep the environment clear.

- If $u(k+p) \leq 10$ the new added workers must not exceed 10, else they will easily cause a safety accident due to the fact that they are not familiar with the environment and a huge amount of technical communications.

Except giving safety training to the workers, sharing enough technical communication, cleaning the operation environment and others are needed. A reasonable worker arrangement must be considered, the following will use MPC to design a more reasonable resource allocation of the workers.

\section{(1) Rolling optimization}

Taken

- $x_{0}=0$ : None initial worker

- $P=4$ : The prediction horizon is 4

- $A=7 ; B=3$ : The weight coefficient in the objective functions

- $\alpha=5$ : A gradient descent coefficient

- $\xi=0.04$ : An iteration calculation stop value.

The iterative steps of the gradient descent method are used to calculate each rolling optimization value, shown in Table 2. 
Table 2. Online optimization (rolling optimization)

\begin{tabular}{|c|c|c|c|c|c|c|c|c|c|c|c|c|}
\hline Value week & W1 & W2 & w3 & w4 & W5 & w6 & w7 & w8 & w9 & w10 & W11 & W12 \\
\hline Reference & 11 & 24 & 33 & 7 & 26 & 19 & 18 & 21 & 37 & 16 & 31 & 25 \\
\hline online 1 & $\underline{9}$ & 19 & 28 & 10 & & & & & & & & \\
\hline online 2 & & $\underline{21}$ & 29 & 10 & 24 & & & & & & & \\
\hline online 3 & & & $\underline{30}$ & 12 & 24 & 20 & & & & & & \\
\hline online 4 & & & & $\underline{13}$ & 24 & 19 & 18 & & & & & \\
\hline online 5 & & & & & $\underline{23}$ & 19 & 18 & 21 & & & & \\
\hline online 6 & & & & & & $\underline{20}$ & 19 & 21 & 34 & & & \\
\hline online 7 & & & & & & & $\underline{18}$ & 21 & 33 & 18 & & \\
\hline online 8 & & & & & & & & $\underline{20}$ & 33 & 18 & 29 & \\
\hline online 9 & & & & & & & & & $\underline{\underline{34}}$ & $\underline{\underline{18}}$ & $\underline{\underline{29}}$ & $\underline{\underline{25}}$ \\
\hline
\end{tabular}

We can see, that if $P=4$, nine iterations are needed to finish all rolling optimizations and every online calculation contains four predict values. And based on MPC theory, the first value will be marked as underlined in the table.

Merging all online predictions output together, Fig. 4 shows a comparing figure between the reference values and MPC online predictive values. From the figure we can see that $\sum_{p=0}^{P-1} B[u(k+p)]^{2}$ makes the control variable more fluent.

\section{MPC Control without Constrait}

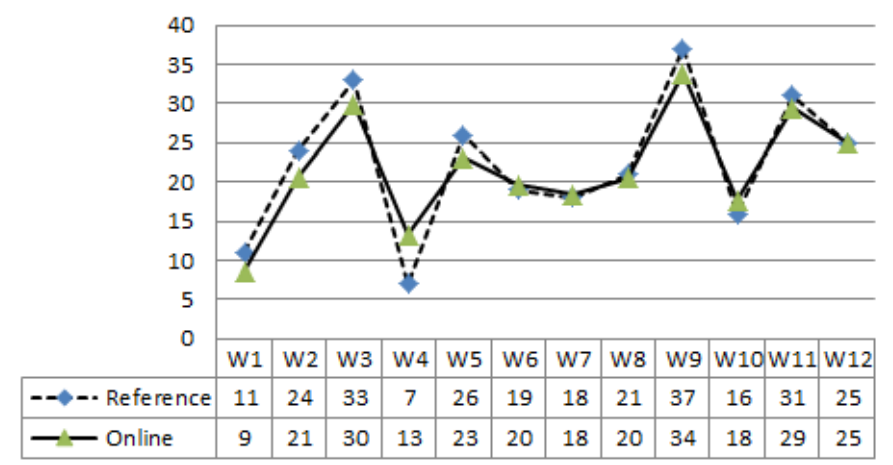

Fig. 4. MPC online control

(2) Feedback correction

Due to the personnel mobility, the real feedback will be different with prediction sometimes. For example, when do "online 9" optimization, the real feedback is 15, not 20. Then the prediction series must be $\hat{x}_{P M}(9)=\{33,17,29,25\}$, not $\hat{x}_{P M}(9)=\{34,18,29,25\}$. Obviously, the rolling optimization is more adaptable to the dynamic system, which is more flexible than offline optimization that shares the optimization values overall offline.

\section{(3) Constraint control}

Another key part of MPC is the constraint control which makes the optimization calculations more complex. We use Microsoft Excel tool to make the 
calculations, a pulsing constraint $\hat{x}(k+p) \leq 30$ and $u(k+p) \leq 10$, then show the calculations in Table 3.

Table 3. Without a constraint vs with a constraint

\begin{tabular}{|c|c|c|c|c|c|c|c|c|c|c|c|c|} 
Value week & W1 & W2 & W3 & W4 & W5 & W6 & W7 & W8 & w9 & W10 & W11 & W12 \\
\hline WithoutC & 9 & 21 & 30 & 13 & 23 & 20 & 18 & 20 & 34 & 18 & 29 & 25 \\
\hline WithC & 9 & 19 & 29 & 12 & 22 & 19 & 18 & 20 & 30 & 17 & 27 & 25 \\
\hline
\end{tabular}

The cell, marked by a dark background has a safety risk, after the new calculation with a constraint. The newest output is acceptable from a safety point of view. Finally, MPC control values with a constraint are given in Fig. 5. We can see that MPC control is more reasonable after considering the safety risk.

\section{MPC Contorl with Constraint}

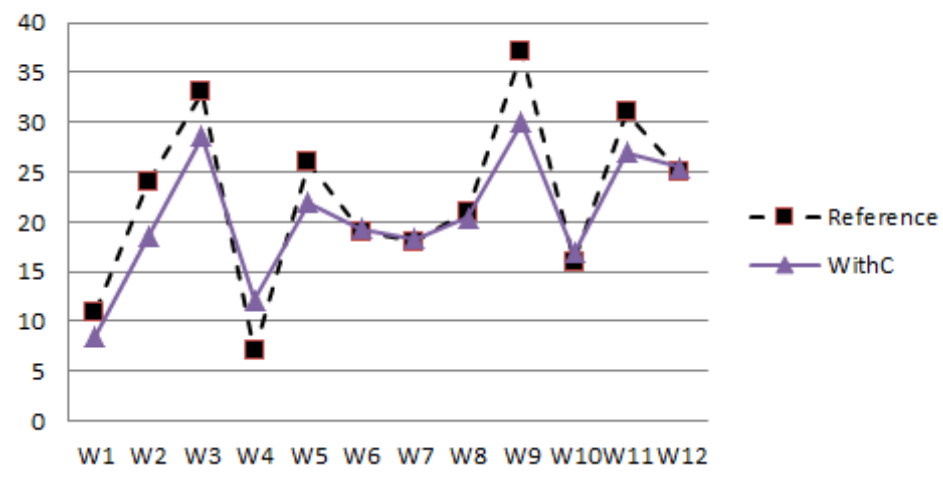

Fig. 5. MPC control with a constraint

\section{Conclusion}

Safety education, following a correct procedure and an engineering method must be applied to reduce the safety risk during the construction of a large scale engineering project. Moreover, more quantitative methods must be considered to give more scientific safety risk control strategy. In this paper, taking the ship-lift engineering construction area in "Three Gorges" project as an example, a valid MPC can be applied into the resource arrangement of LSE. The numeric calculation results show how rolling optimization, feedback correlation and constraint control work in a MPC model which is generally a secret part due to commercial purposes.

This paper is a meaningful research to apply MPC theory into safety risk management of large-scale engineering. The safety risk of a large-scale engineering system is a dynamic complex procedure affected by many factors. Consequently, it may be extremely difficult to construct an accurate and complete mathematical model for the system. However, MPC has this advantage that it does not require an accurate mathematical model, as long as the model can be predicted. In fact, the 
safety risk issue is an optimization issue, a feedback deviation issue, a constraint issue and a predictive issue. This supposes that MPC must have appropriate further development in safety risk management.

Acknowledgement: The paper is sponsored by the Chinese National Natural Science Foundation Project (71271031): "The early warning index system construction and controlling of safety risk based on optimal control theory for large-scale engineering with empirical research" and the Chinese National Natural Science Foundation Project (70972123): “The organizational knowledge integration optimization management and empirical research about the hall for workshop of meta synthetic engineering in the process management of mega-scale engineering under complex and dynamic environment”.

\section{References}

1. L e e la, D a modaran, R. H. J orgen, M. H. Tarek. Impact of Large Scale Engineering Products and Processes on Society - The eLSEwise View. - Engineering, Construction and Architectural Management, Vol. 6, 1999, No 1, 63-70.

2. Thielman, J., Ge Ping. Applying Axiomatic Design Theory to the Evaluation and Optimization of Large-Scale Engineering Systems. - Journal of Engineering Design, Vol. 17, 2006, No 1, 1-16.

3. Wail, Menesi, Golzarpoor Behrooz, Hegazy Tarek. Fast and Near-Optimum Schedule Optimization for Large-Scale Projects. - Journal of Construction Engineering \& Management, Vol. 139, 2013, No 9, 1117-1124.

4. Enno, Koehn, K. Datta Nirmal. Quality, Environmental, and Health and Safety Management Systems for Construction Engineering. - Journal of Construction Engineering \& Management, Vol. 129, 2003, No 5, 562-569.

5. Gu o, H. L., L i Hen g, L i V e r a. VP-Based Safety Management in Large-Scale Construction Projects: A Conceptual Framework. - Automation in Construction, Vol. 34, 2013, 16-24.

6. J u n, D h o H e o n, E l-R a y e s, Kh a l e d. Fast and Accurate Risk Evaluation for Scheduling Large-Scale Construction Projects. - Journal of Computing in Civil Engineering, Vol. 25, 2011, No 5, 407-417.

7. J i a n f e n g, Q i a o. Resource Allocation of Large Scale Engineering Based on Optimal Control Theory. - International Journal of Control \& Automation, Vol. 7, 2014, No 1, 199-214.

8. Brdys, Mietek A., Vu Nam Tran, Wojtek Kurek. Safety Zones Based Robustly Feasible Model Predictive Control for Nonlinear Network Systems. - In: Proc. of International Federation of Automatic Control World Congress, Milano, Italy, 2011, 4821-4826.

9. Torste in, I. B., A. J. T or. Dynamic Safety Constraints by Scenario Based Economic Model Predictive Control.

10. J ohn, M. C., B e h c et A c i kme se, M. M. Ri chard, G. M. D o u gla s. Robust Model Predictive Control Algorithm with a Reactive Safety Mode. - Automatica, Vol. 49, 2013, No 5, 1251-1260.

11. Kettunen, M., P. Zhang, S. L. J. Jounela, M. Kettunen, P. Zhang, S. L. J. J o u n e la. An Embedded Fault Detection, Isolation and Accommodation System in a Model Predictive Controller for an Industrial Benchmark Process. - Computers \& Chemical Engineering, Vol. 32, 2008, No 12, 2966-2985.

12. Fe ifei, Lui. Difficulties from Safety Management of Construction of Ship-Lift for Three Gorges Project and Relevant Control Measures. - Water Resources and Hydropower Engineering, Vol. 44, 2013, No 4, 52-56. 